J.M. Burton, MD, MSc, FRCPC

S. Kimball, MSc, MLT

R. Vieth, PhD

A. Bar-Or, MD, MSc, FRCPC

H.-M. Dosch, MD, PhD

R. Cheung, MSc

D. Gagne

C. D'Souza, PhD

M. Ursell, MS, MSc, FRCPC

P. O'Connor, MS, MSc, FRCPC

Address correspondence and reprint requests to Dr. Jodie $M$. Burton, Department of Clinical Neurosciences, Rockyview General Hospital, 5th Floor, Room 5A214, 7007 14th Street SW, Calgary, Alberta T2V 1P9, Canada

jodie.burton@albertahealthservices.ca

Editorial, page 1846

Supplemental data at www.neurology.org

\section{A phase I/II dose-escalation trial of vitamin D3 and calcium in multiple sclerosis}

\section{소}

\section{ABSTRACT}

Objective: Low vitamin D status has been associated with multiple sclerosis (MS) prevalence and risk, but the therapeutic potential of vitamin D in established MS has not been explored. Our aim was to assess the tolerability of high-dose oral vitamin D and its impact on biochemical, immunologic, and clinical outcomes in patients with MS prospectively.

Methods: An open-label randomized prospective controlled 52-week trial matched patients with MS for demographic and disease characteristics, with randomization to treatment or control groups. Treatment patients received escalating vitamin D doses up to $40,000 \mathrm{IU} /$ day over 28 weeks to raise serum 25-hydroxyvitamin D [25(OH)D] rapidly and assess tolerability, followed by 10,000 IU/day (12 weeks), and further downtitrated to $0 \mathrm{IU} /$ day. Calcium $(1,200 \mathrm{mg} /$ day $)$ was given throughout the trial. Primary endpoints were mean change in serum calcium at each vitamin $D$ dose and a comparison of serum calcium between groups. Secondary endpoints included 25(OH)D and other biochemical measures, immunologic biomarkers, relapse events, and Expanded Disability Status Scale (EDSS) score.

Results: Forty-nine patients (25 treatment, 24 control) were enrolled [mean age 40.5 years, EDSS 1.34, and 25(OH)D $78 \mathrm{nmol} / \mathrm{L}$. All calcium-related measures within and between groups were normal. Despite a mean peak 25(OH)D of $413 \mathrm{nmol} / \mathrm{L}$, no significant adverse events occurred. Although there may have been confounding variables in clinical outcomes, treatment group patients appeared to have fewer relapse events and a persistent reduction in T-cell proliferation compared to controls.

Conclusions: High-dose vitamin D ( 10,000 IU/day) in multiple sclerosis is safe, with evidence of immunomodulatory effects.

Classification of evidence: This trial provides Class II evidence that high-dose vitamin D use for 52 weeks in patients with multiple sclerosis does not significantly increase serum calcium levels when compared to patients not on high-dose supplementation. The trial, however, lacked statistical precision and the design requirements to adequately assess changes in clinical disease measures (relapses and Expanded Disability Status Scale scores), providing only Class level IV evidence for these outcomes. Neurology ${ }^{\circledR}$ 2010;74:1852-1859

\section{GLOSSARY}

ALP = alkaline phosphatase; $\mathbf{A L T}=$ alanine aminotransferase; $\mathbf{A S T}=$ aspartate aminotransferase; $\mathbf{E A E}=$ experimental autoimmune encephalitis; EDSS = Expanded Disability Status Scale; IL = interleukin; LS = least squares; MMP-9 = matrix metalloproteinase-9; $\mathbf{M S}=$ multiple sclerosis; $\mathbf{P T H}=$ parathyroid hormone; TCS = T-cell score; TIMP-1 = tissue inhibitory of metalloproteinase-1; TNF $\boldsymbol{\alpha}=$ tumor necrosis factor- $\alpha$.

Multiple sclerosis (MS) has a well-documented geographic distribution, with increasing prevalence and risk with increasing distance from the equator. ${ }^{1-4}$ Limited sunlight and UVB exposure, MS risk factors based on observational studies, are intermediaries between latitude and MS. ${ }^{2-5}$ Low serum $25(\mathrm{OH}) \mathrm{D}$ also appears to be a risk factor, and is a direct product of skin exposure to UVB. ${ }^{4-7}$

e-Pub ahead of print on April 28, 2010, at www.neurology.org.

From the Division of Neurology (J.M.B., P.O.), St. Michael's Hospital, Toronto; University of Toronto (J.M.B., S.K., R.V., H.-M.D., R.C., C.D., P.O.), Toronto; Department of Nutritional Sciences and Laboratory Medicine and Pathology (S.K., R.V.), Mount Sinai Hospital, Toronto; Montreal Neurological Institute (A.B.-O., D.G.), Montreal; McGill University (A.B.-O., D.G.), Montreal; The Neurosciences \& Mental Health Program (H.-M.D., R.C.), Hospital for Sick Children, Toronto; Center for Research in Neurodegenerative Diseases (C.D.), University of Toronto; and Division of Neurology (M.U.), Etobicoke General Hospital, Toronto, Canada.

Study funding: Supported by peer-reviewed grants from Direct-MS Charity and from the MS Society of Canada.

Disclosure: Author disclosures are provided at the end of the article. 
Patients with MS have relatively low serum $25(\mathrm{OH}) \mathrm{D}$, with evidence that disease activity may increase when UVB exposure is limited..$^{8-10}$ Additionally, increased sun exposure in early life significantly reduces disease development. ${ }^{5}$

In studies of vitamin D and calcium in experimental autoimmune encephalitis (EAE), preinduction treatment prevents disease development, while postinduction treatment ameliorates disease activity. ${ }^{11}$ Vitamin $\mathrm{D}$ has antiinflammatory actions in vitro, including enhanced Th2 and decreased Th1 cytokine production, dendritic cell effects, and enhanced macrophage phagocytosis. ${ }^{12}$

Vitamin D clearly has utility in MS prevention, but its role in established MS remains unknown. Observational studies have shown patients with MS respond to vitamin $\mathrm{D}$ and sun exposure. . $-6,8-10,12$ Physiologically, serum $25(\mathrm{OH}) \mathrm{D}$ acquired through sun exposure ranges up to $220 \mathrm{nmol} / \mathrm{L}$ with no recognized toxicity. ${ }^{13}$ Furthermore, patients with MS tolerated a pilot dose-escalation trial up to 40,000 IU/day. ${ }^{14}$

The present trial was undertaken to establish tolerability of high-dose vitamin $\mathrm{D}$ with calcium in a larger sample of patients with MS over 52 weeks. The hypothesis was that such doses are safe as measured by serum calcium over the dosing regimen. Secondary outcomes included serum parathyroid hormone (PTH), $25(\mathrm{OH}) \mathrm{D}$, creatinine, transaminases, urinary calcium, relapses, disability (Expanded Disability Status Scale [EDSS]), T-cell reactivity, cytokines, and matrix-metalloproteinases.

METHODS Standard protocol approvals, registrations, and patient consents. The trial was approved by the institutional review boards at St. Michael's Hospital (University of Toronto, Toronto, Canada) and McGill University (Montreal, Canada) and Health Canada for experimentation in human subjects. The trial was also registered with Clinicaltrials.gov through the National Institute of Health (ID NCT00644904). All patients provided written informed consent and were enrolled by the primary author.

Enrollment was limited to Multiple Sclerosis Clinic patients at St. Michael's Hospital between June 2006 and March 2007. with the last patient completing the 52-week trial in March 2008. All patients were asked if they were interested in participating in any of the trials at the center for which they were eligible by clinic physicians. If patients were interested in the vitamin D trial, authors Dr. Burton or S. Kimball would speak with the patients at their request. Relapse assessments were performed by authors Dr. Burton and Dr. O'Connor.

\begin{tabular}{|c|c|c|c|}
\hline \multirow{3}{*}{$\begin{array}{l}\text { Table } 1 \\
\text { Trial visit }\end{array}$} & \multicolumn{3}{|c|}{$\begin{array}{l}\text { Treatment group vitamin D3 and } \\
\text { calcium dosing schedule }\end{array}$} \\
\hline & \multirow[b]{2}{*}{ Trial week } & \multicolumn{2}{|c|}{ Supplementation } \\
\hline & & $\begin{array}{l}\text { Vitamin D3 } \\
\text { (IU/wk) }\end{array}$ & $\begin{array}{l}\text { Calcium } \\
\text { (mg/d) }\end{array}$ \\
\hline 1 & 1 & 0 & 1,200 \\
\hline 2 & 3 & 28,000 & 1,200 \\
\hline 3 & 5 & 70,000 & 1,200 \\
\hline 4 & 11 & 112,000 & 1,200 \\
\hline 5 & 17 & 224,000 & 1,200 \\
\hline 6 & 23 & 280,000 & 1,200 \\
\hline 7 & 29 & 70,000 & 1,200 \\
\hline 8 & 35 & 70,000 & 1,200 \\
\hline 9 & 41 & 28,000 & 1,200 \\
\hline 10 & 49 & 0 & 0 \\
\hline 11 & 52 & 0 & 0 \\
\hline
\end{tabular}

Patients were eligible for inclusion if they were $18-55$ years of age with clinically definite MS according to McDonald criteria and an EDSS score of 0-6.5. ${ }^{15,16}$ Patients could continue with their MS disease-modifying medication while in the trial, the monitoring of which was performed by authors Dr. Burton and Dr. O'Connor. Patients with the following were excluded: relapse within 60 days, steroid use within 30 days, chemotherapy within 12 months, pregnancy/inadequate contraception, vitamin $\mathrm{D}$ intake $>4,000 \mathrm{IU} /$ day, serum $25(\mathrm{OH}) \mathrm{D}$ level $>150$ $\mathrm{nmol} / \mathrm{L}$, lymphoma, granulomatous disease, cardiac arrhythmia, kidney dysfunction, or disordered calcium metabolism.

Trial design and outcomes. This was a phase I/II randomized open-label trial focusing on vitamin $\mathrm{D}$ safety. This design provides Class II level evidence for the primary outcome of serum calcium values across the treatment dosing regimen.

Patients were matched for age (18-30 years, 31-40 years, $41-55$ years), disease duration ( $0-9$ years, $10-19$ years, $\geq 20$ years), EDSS ( $\leq 2.5,3.0-5.5,6.0-6.5)$, and disease-modifying drug (interferon, glatiramer acetate, none). Members of pairs were randomized to treatment or control groups based on blinded drawing from a hat.

Treatment patients began calcium supplementation $(1,200$ $\mathrm{mg}$ ) at visit 1 and initiated vitamin D 2 weeks later following the dosing regimen shown in table 1 . Control patients were permitted to take $\leq 4,000 \mathrm{IU} /$ day of vitamin $\mathrm{D}$ and supplemental calcium if desired.

All patients had EDSS and general medical examinations at baseline and end of the trial. Serum calcium, albumin, $25(\mathrm{OH}) \mathrm{D}$, $\mathrm{PTH}$, transaminases (alanine aminotransferase [ALT], aspartate aminotransferase $[\mathrm{AST}]$, alkaline phosphatase $[\mathrm{ALP}])$, creatinine, urea and urinary calcium/creatinine ratios were measured in treatment patients at all visits. Treatment patients were seen every 6 weeks for the above testing and to obtain subsequent doses of vitamin D. Renal ultrasounds to monitor for calcifications were performed at baseline and weeks 29 and 52, while EKGs were performed at baseline and week 52 . Serum was drawn and cryopreserved for cytokine analysis and matrix metalloproteinase-9 (MMP9)/tissue inhibitory of metalloproteinase-1 (TIMP-1) in all patients while fresh blood was drawn at baseline and week 52 for T-cell assays. Control patients had $25(\mathrm{OH}) \mathrm{D}$ and calcium/albumin levels and T-cell reactivity testing at weeks 1 and 52, while MMP-9/ 
TIMP-1 and cytokines were assessed at weeks 1, 11, 29, and 52 (see table e-1 on the Neurology ${ }^{\circledR}$ Web site at www.neurology.org for schedule).

Patients experiencing relapse (defined as an objective new/ reemerging neurologic abnormality present for at least 24 hours in the absence of fever/infection) or adverse events were seen within 72 hours by the treating physician. Patients experiencing relapse events received steroids (IV methylprednisolone or oral prednisone) as deemed appropriate by the treating physician. All events were documented on adverse event reporting forms.

Vitamin D3 preparation and administration. The preparation and quality testing of ethanol solutions of vitamin $\mathrm{D}$ has been previously described. ${ }^{14}$ Hyperconcentrated solutions were supplied in amber glass vials at every dose-changing visit. $\mathrm{Pa}$ tients were instructed to take anywhere between 1 and $4 \mathrm{~mL} / \mathrm{wk}$ orally (depending on that week's concentration) to achieve the desired dose, but only after serum calcium and urinary calcium/ creatinine measured at that time were found to be within normal limits. Patients also received tricalcium phosphate powder and were instructed to take a measured amount equivalent to $1,200 \mathrm{mg} /$ day by mouth. Consistent failure of $25(\mathrm{OH}) \mathrm{D}$ to increase with increasing dose was taken as evidence of possible noncompliance.

\begin{tabular}{|c|c|c|c|}
\hline Patient den & aphics at baseline & & \\
\hline Variable & Treatment group & Control group & p Value \\
\hline \multicolumn{4}{|l|}{ Age, y } \\
\hline Mean (range) & $41.1(22-54)$ & 39.9 (21-53) & - \\
\hline SD (95\% Cl) & 7.4 (38.0-44.2) & $8.6(36.3-43.5)$ & \\
\hline Gender, F/M & $21 / 4$ & $19 / 5$ & - \\
\hline MS subtype, RR/SP & $23 / 2$ & $22 / 2$ & - \\
\hline \multicolumn{4}{|l|}{ Disease duration, y } \\
\hline Mean (range) & $8.2(1-25)$ & $7.4(1-21)$ & - \\
\hline SD $(95 \% \mathrm{Cl})$ & $6.7(5.4-10.9)$ & $6.0(4.8-9.9)$ & \\
\hline \multicolumn{4}{|l|}{ Baseline EDSS } \\
\hline Mean (range) & $1.46(0-6.0)$ & $1.23(0-6.0)$ & - \\
\hline SD $(95 \% \mathrm{Cl})$ & $1.55(0.82-2.10)$ & $1.63(0.54-1.92)$ & \\
\hline $\begin{array}{l}\text { Season enrolled, spring or } \\
\text { summer/fall or winter }\end{array}$ & $16 / 9$ & $11 / 13$ & NS \\
\hline \multicolumn{4}{|l|}{ Annualized relapse rate } \\
\hline Mean (range) & $0.44(0-3)$ & $0.54(0-2)$ & NS \\
\hline SD $(95 \%$ Cl) & $0.77(0.12-0.76)$ & $0.72(0.24-0.84)$ & \\
\hline DMD use, IFN/GA/NO & $12 / 2 / 11$ & $12 / 2 / 10$ & NS \\
\hline \multicolumn{4}{|l|}{ Vitamin D3 intake, IU/d } \\
\hline Mean (range) & $1,160(0-4,000)$ & $991(0-4,000)$ & NS \\
\hline SD $(95 \% \mathrm{Cl})$ & $1,489(545-1,774)$ & $1,334(427-1,554)$ & \\
\hline \multicolumn{4}{|l|}{ Serum 25(OH)D, nmol/L } \\
\hline Mean (range) & $73(38-146)$ & $83(38-154)$ & NS \\
\hline SD $(95 \% \mathrm{Cl})$ & 26 (62-83) & $27(71-94)$ & \\
\hline \multicolumn{4}{|l|}{ Serum calcium, mmol/L } \\
\hline Mean (range) & $2.32(2.08-2.57)$ & $2.32(2.16-2.47)$ & NS \\
\hline SD (95\% Cl) & $0.10(2.28-2.37)$ & $0.08(2.28-2.35)$ & \\
\hline
\end{tabular}

Abbreviations: $\mathrm{Cl}=$ confidence interval; $\mathrm{DMD}=$ disease-modifying medication; EDSS = Expanded Disability Status Scale; GA = glatiramer acetate; IFN = interferon beta; MS = multiple sclerosis; $\mathrm{NO}=$ none; $\mathrm{RR}=$ relapsing-remitting; $\mathrm{SP}=$ secondary progressive.
Immunologic methods. Peripheral blood T-cell proliferative responses to a panel of 13 MS-related and dietary antigens and 4 control test antigens were measured as previously described. ${ }^{17}$ For comparisons over time and among groups, a T-cell score (TCS) was generated from the number of positive responses to these antigens.

Serum levels of matrix metalloproteinases (MMP-9 and TIMP-1) were measured with the Quantikine ELISA system (R\&D Systems Inc., Minneapolis, MN) according to manufacturer's directions. MMP-9, TIMP-1, and MMP-9/TIMP-1 were evaluated at weeks $1,11,29$, and 52 in all patients.

Cytokine samples were drawn from serum and from the supernatant of the T-cell proliferation assays at baseline and end of trial in all patients, and in the treatment group patients at weeks 2 and 11 to reduce statistical error. Cytokine concentrations (interleukin [IL]-1 $\beta$, IL-2, IL-4, IL-5, IL-6, IL-10, IL-12p40, IL13 , interferon- $\gamma$, and tumor necrosis factor- $\alpha$ [TNF $\alpha]$ ) were measured simultaneously by multiplex bead immunoassays and read on the Luminex LX100 as previously described. ${ }^{18}$

Statistical analysis. All analyses assumed a 2-sided test of hypothesis with an overall $\alpha$ value of 0.05 . Immunologic end points and post hoc analyses, which included all time points, were considered exploratory and were not adjusted for multiple comparisons. Descriptive statistics were presented as means with SD. Nonparametric tests were used to compare characteristics between treatment groups.

Sample size was based on a power of 0.90 at a 2 -sided 0.05 significance level, if the true difference between the treatment and control groups was $1 \mathrm{SD}$. This would have been a clinically meaningful increase in the calcium-related measures most likely to be adversely affected by the vitamin $\mathrm{D}$ including renal and hepatic markers. A sample of 60 patients provided $>0.90$ power with an $\alpha$ of 0.05 for the primary meaningful effect size of a 0.30 $\mathrm{mmol} / \mathrm{L}$ difference in serum calcium ( $\alpha$ of 0.05 ).

For outcomes, sign/sign rank tests were used to evaluate continuous outcomes. McNemar testing was used to analyze categorical and binary outcomes while repeated measures were calculated using mixed modeling procedures and time series analysis. For nonparametric testing on unpaired continuous data, the Wilcoxon rank sum test was used. All statistical tests were performed using SAS 9.1.3 $3^{\mathrm{TM}}$.

RESULTS Between June 2006 and March 2007, 51 patients were screened and 49 matched for age, disease duration, EDSS, and disease-modifying drug (25 treatment, 24 control). Due to feasibility constraints, enrollment was stopped at 51 subjects, but this still provided $>0.90$ power for the primary endpoint and was approved by the institutional review boards at participating centers. Baseline demographics are outlined in table 2. Three subjects did not have exact matches. Forty-five patients had relapsingremitting MS and 4 had secondary progressive MS. Forty women and 9 men participated. The mean age of all subjects was 40.5 years (21-54), with mean EDSS $1.34(0-6.0)$ and disease duration of 7.8 years (1-25). At trial entry, mean baseline calcium was $2.32 \mathrm{mmol} / \mathrm{L}(2.08-2.57)$ and mean was $25(\mathrm{OH}) \mathrm{D}$ $78 \mathrm{nmol} / \mathrm{L}(38-154)$. There were no differences between groups for these or any baseline characteristics 


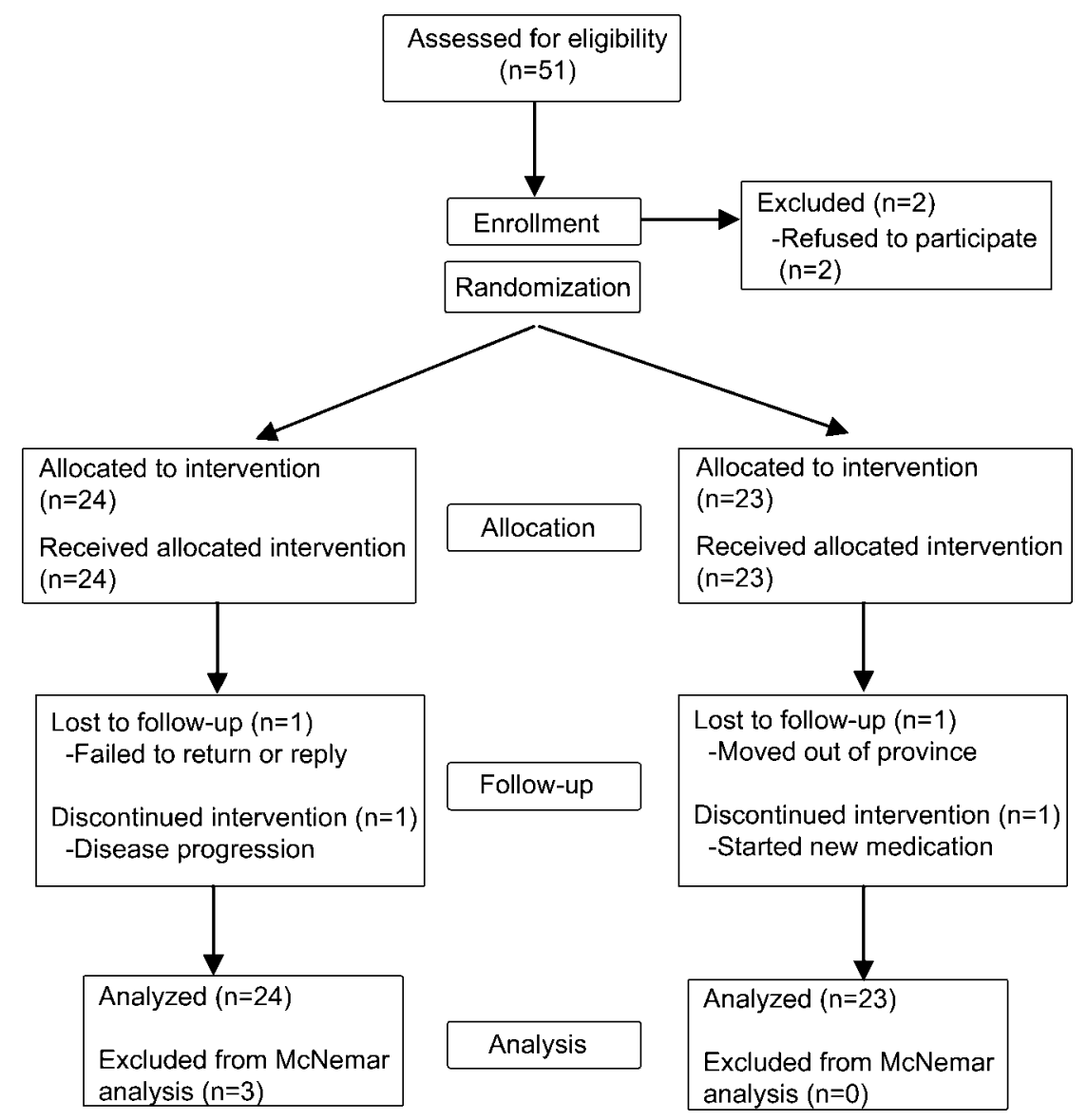

including pretrial vitamin $\mathrm{D}$ intake and relapse rate. Two patients in each group withdrew during the trial, none related to adverse events (figure 1).

Safety and biochemical outcomes. The primary outcome, serum calcium at each dose in the treatment regimen, was evaluated by repeated measures/mixed modeling procedures. The influence of season was ruled out using time series analysis. No significant difference in serum calcium occurred at any point along the dosing regimen, nor did any serum calcium values exceed the upper limit of the reference range (2.10-2.60 mmol/L) (figure 2, A and B).

Urinary calcium was evaluated in a similar manner, and during the weeks at the highest doses of vitamin D (weeks 17-29), urinary calcium/creatinine ratios rose to a maximum mean of 0.61 (mixed modeling least squares [LS] means, $p=0.014$ ), but always below the cutoff of 1.0 (figure $2 \mathrm{C}$ ). PTH varied significantly over the trial, but within the normal range $(1.3-5.4 \mathrm{pmol} / \mathrm{L})$ and not influenced by vitamin D dose (mixed modeling LS means, $p=0.02$ ) (table e-2).

As expected, serum $25(\mathrm{OH}) \mathrm{D}$ rose considerably, reaching a maximum mean of $413 \mathrm{nmol} / \mathrm{L}$ following the 40,000 IU/day dosing period. This value is well above the cited "toxic" value of $250 \mathrm{nmol} / \mathrm{L} .{ }^{13} \mathrm{In}$ fact, patient mean serum $25(\mathrm{OH}) \mathrm{D}$ values were above this threshold for over 18 weeks with no adverse consequences (figure $2 \mathrm{D}$ ).

Renal and hepatic function was assessed by creatinine/urea and ALT/AST/ALP at all visits in the treatment group. There were no cases of disordered creatinine, urea, ALT, AST, or ALP (table e-2).

No patient had evidence of renal calcification on ultrasound. Incidental chronic pelviectasis was found in one asymptomatic patient without nephrolithiasis. Another patient had preexisting chronic hemangiomas while a third had transient fatty liver attributed to recent steroid therapy. No disturbances of cardiac rhythm were seen.

Clinical outcomes. As this trial was unblinded, all clinical results are exploratory and subject to bias. Mean annualized relapse rate in treatment patients decreased from 0.44 to 0.26 , while in control patients, it also decreased, from 0.54 to 0.45 , although this was not significant by sign test (table 3 ). The proportion of treatment patients experiencing relapses during the trial was 0.16 vs 0.37 in the control group (McNemar, $p=0.09$ ). Some trends favoring the treatment group were seen in EDSS measures, 

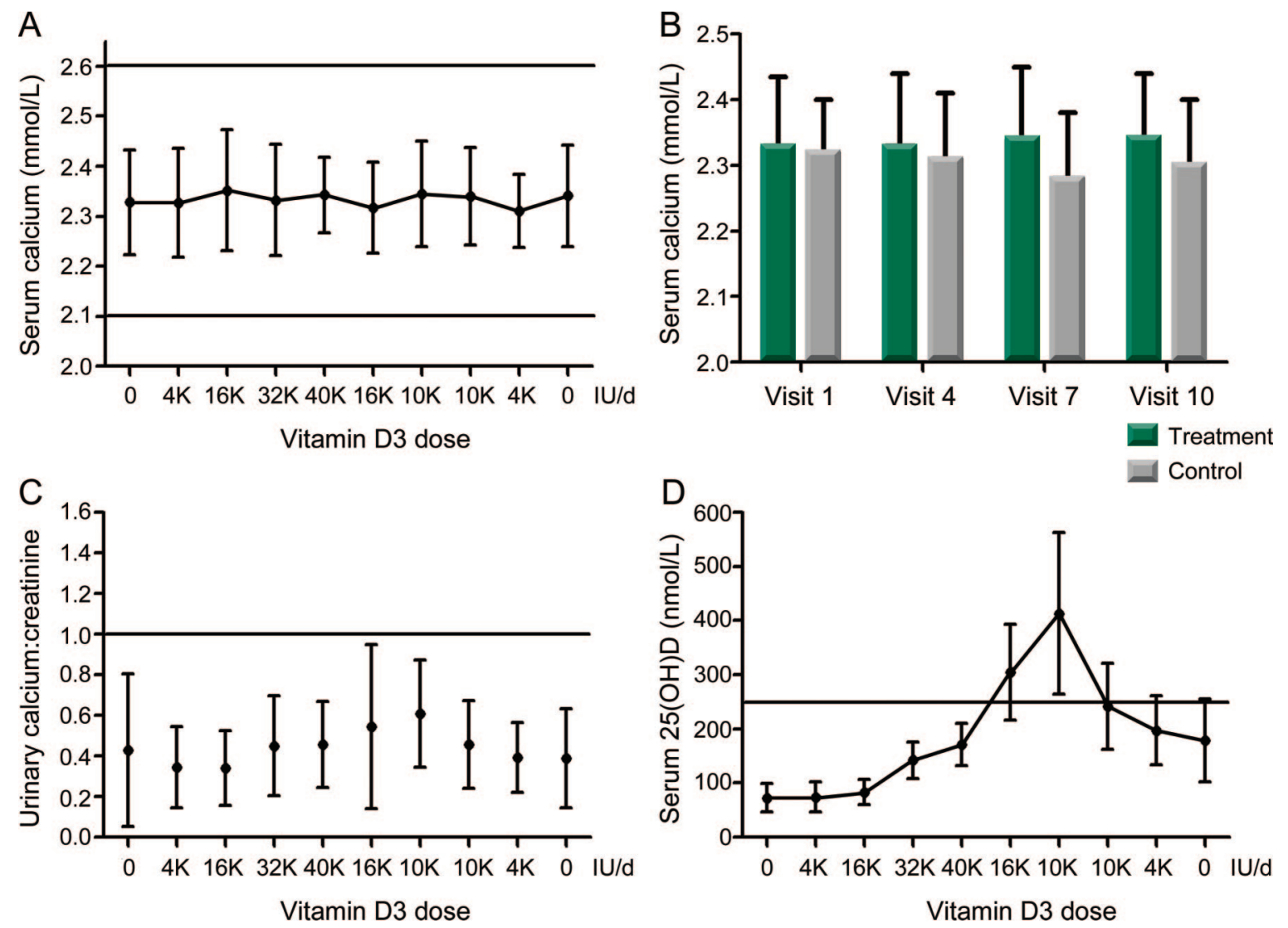

(A) Mean serum calcium (mmol/L) over dosing regimen in treatment group. (B) Mean serum calcium (mmol/L) of control and treatment groups at week 1 (O IU/day vitamin D3 for treatment group), week (10,000 IU/day of vitamin D3 for treatment group), week (40,000 IU/day of vitamin D3 for treatment group), and week (discontinuation of vitamin D3 for treatment group). (C) Urinary calcium: creatinine ratios over dosing regimen in treatment group. (D) Serum 25(OH)D (nmol/L) over dosing regimen in treatment group [the value on the $y$-axis reflects the mean 25(OH)D level after completing the course associated with the dose of vitamin D on the $x$-axis].

but the majority of patients had low EDSS scores throughout the trial with changes typically of 0.5 to 1 EDSS point (table 3).

Immunologic outcomes. Lymphocyte response assays. T-cell proliferative responses were compared at the first and final visits, 52 weeks apart, in all patients. The total TCS, a tally of the number of positive proliferative responses over the trial in response to antigenic stimulation, decreased significantly in the treatment group (sign test, $p=0.002$ ), with no change in controls. The proportion of patients who completed the trial with a total TCS below the predetermined threshold of 4 was greater in the treatment group (McNemar, $p=0.052$ ). When comparing those who completed the trial with a $25(\mathrm{OH}) \mathrm{D}$ level $=>100 \mathrm{nmol} / \mathrm{L}$ to those with values $<100 \mathrm{nmol} / \mathrm{L}$, a significantly larger proportion of those with decreased TCS was seen in those with levels $\geq 100 \mathrm{nmol} / \mathrm{L}$ (Wilcoxon rank sum test, $p=$ 0.032).

MMP-9 and TIMP-1. Repeated measurements of MMP-9 and its inhibitor TIMP-1 over 4 time points during the trial revealed small reductions between visits over the course of the trial in both treatment and control groups. Neither magnitude nor direction of change differed significantly between groups.

Cytokine profile. Cytokine measures were carried out in duplicate, and the mean of these measures was used in all subsequent analyses. Analysis for IL-1 $\beta$, IL-2, IL-4, IL-5, IL-6, IL-10, IL-12p40, IL-13, interferon- $\gamma$, and TNF $\alpha$ was carried out in both serum samples collected at the indicated time points, as well as in supernatants of the lymphocyte response assay samples described above. There were no consistent patterns of change in cytokine titers within or between groups over the 52-week period (data not shown).

A separate report by this group on the immunologic outcomes is currently in preparation.

Adverse events. No adverse events with the use of vitamin D were reported. Four treatment patients experienced mild constipation early in the trial attributed to calcium supplementation in powdered 
Table $3 \quad$ Clinical outcomes ${ }^{a}$

\begin{tabular}{|c|c|c|c|}
\hline Outcome & Treatment $(n=25)$ & Control $(n=24)$ & $\begin{array}{l}\text { p Value } \\
(95 \% \mathrm{Cl})\end{array}$ \\
\hline ARR year prior to trial (mean, $95 \% \mathrm{Cl}$ ) & $0.44(0.77 ; 0.12$ to 0.76$)$ & $0.54(0.72 ; 0.24$ to 0.84$)$ & $N S^{b}$ \\
\hline ARR during trial year (mean, $95 \% \mathrm{Cl}$ ) & $0.26(0.62 ;-0.06$ to 0.53$)$ & $0.45(0.59 ; 0.19$ to 0.72$)$ & 0.09 \\
\hline \multirow{2}{*}{$\begin{array}{l}\text { Change in ARR from year } \\
\text { prior to year on trial (just \% change) }\end{array}$} & $-41 \%$ & - & $0.17( \pm 0.18)^{b}$ \\
\hline & - & $-17 \%$ & $0.43( \pm 0.36)^{b}$ \\
\hline Proportion with relapses & 0.16 & 0.37 & $0.09^{c}$ \\
\hline End of trial EDSS (mean, 95\% Cl) & $1.15(1.39 ; 0.55$ to 1.75$)$ & $1.45(1.78 ; 0.66$ to 2.24$)$ & $N S^{b}$ \\
\hline Change in EDSS over trial (just \% change) & -0.23 & +0.30 & $N S^{b}$ \\
\hline $\begin{array}{l}\text { Proportion completing trial } \\
\text { with increased EDSS }\end{array}$ & 0.08 & 0.375 & $0.019^{c}$ \\
\hline
\end{tabular}

Abbreviations: ARR = annualized relapse rate; $\mathrm{Cl}=$ confidence interval; EDSS = Expanded Disability Status Scale. ${ }^{a}$ Intention to treat used for all analyses with the exception of McNemar testing, which requires only those within a matched pair be included. $p$ Values $\geq 0.50$ are not significant. Values are (standard deviation; $95 \%$ confidence interval).

b Sign/sign rank test.

${ }^{c}$ McNemar test.

form. All symptoms resolved with calcium formulation change or discontinuation.

DISCUSSION We have demonstrated that highdose oral vitamin $\mathrm{D}$ in patients with MS, at doses resulting in serum $25(\mathrm{OH}) \mathrm{D}$ concentrations approximately double the physiologic rage obtainable by UV exposure, did not result in clinical, biochemical, or radiologic adverse events. ${ }^{13}$ Neither hypercalcemia nor persistent hypercalciuria occurred, nor did any disruption in renal or hepatic function. Treated patients spent 36/52 weeks on doses of 10,000 IU/day of VD3 or greater, with a mean daily dose of roughly $14,000 \mathrm{IU} /$ day, reaching a mean peak of serum $25(\mathrm{OH}) \mathrm{D}>400 \mathrm{nmol} / \mathrm{L}$.

The trial was not powered nor blinded to properly address clinical outcomes, but we did observe that clinical outcomes appeared to favor the treatment group.

T-cell reactivity and proliferation dropped significantly in treatment patients over the 52-week period, while no change was seen in controls. This decrease was most pronounced in patients who maintained a $25(\mathrm{OH}) \mathrm{D}$ level $\geq 100 \mathrm{nmol} / \mathrm{L}$ at 52 weeks. While long-term treatment of larger cohorts is required to seek relationships between clinical course improvements and T-cell reactivity, the latter observation may provide a first, tentative target range for therapeutic serum $25(\mathrm{OH}) \mathrm{D}$ levels in patients with MS. Unfortunately, measurement of more surrogate markers of proinflammatory and antiinflammatory markers in serum and T-cell culture supernatants disappointed. MMP-9/TIMP-1 values changed over the trial, but the nature and magnitude of change did not differ between groups, and cytokine profiles did not yield meaningful patterns of change under trial conditions. The explanation for these outcomes may ultimately be the true lack of impact of VD3 on such markers or confounding supplementation use with vitamin $\mathrm{D}$ or other agents in controls, but may also be related to methodologic limitations including power, regression to the mean, or degradation of sample integrity after prolonged cryopreservation. Additionally, changes in these markers may occur over much briefer time periods or to a much smaller extent than could be detected, and were missed.

Unlike earlier trials using low-dose vitamin D or calcitriol in MS, our trial used high-dose vitamin D (the direct product of UVR exposure of human skin), and was the first controlled trial to test the safety of such high doses over an extended period of time. ${ }^{19,20}$ A major strength was the novel titration schedule used, minimizing the risk of toxicity at higher doses because doses were only increased after the safety of preceding doses was confirmed. As well, the addition of calcium supplementation allowed safety data to be generalized to those already on calcium supplementation, and the combination of vitamin D and calcium appears most effective in both EAE models and human cancer prevention trials. ${ }^{11,18}$ The present trial's sample size provided sufficient power for primary endpoints of safety measures, namely serum calcium levels, and the use of vitamin D (cholecalciferol) provided additional safety from hypercalcemia, as this inactive precursor of the hormonal metabolite, calcitriol, has low calcemic potential. ${ }^{19,20}$ An earlier trial of calcitriol in MS led to several instances of hypercalcemia. ${ }^{19,20}$

Our trial, however, is not without limitations. The matching design limited patient enrollment and recruitment duration, and selection and volunteer 
bias cannot be ruled out. Although the primary outcomes (serum calcium values) were not vulnerable to a lack of blinding, clinical outcomes certainly may have been. More frequent monitoring of the treatment group could have been a factor in the disclosure of relapses and may have missed possible events or use of undisclosed agents in the less frequently assessed control group. ${ }^{21}$ Furthermore, changes in EDSS were admittedly typically no greater than 0.5 to 1 point, and in a group with a relatively low EDSS at baseline. Additionally, while more robust results may have been seen had the control group not taken any vitamin $\mathrm{D}$, such restrictions were deemed unethical.

We have demonstrated that vitamin $\mathrm{D}$ intake well above current recommendations, and $25(\mathrm{OH}) \mathrm{D}$ levels beyond the physiologic range, do not expose patients with MS to adverse biochemical or clinical events. Compared to a control group whose intake of vitamin D generally exceeded the current North American recommendations, only those on the treatment regimen had evidence of immunologic effects. Whether toxicity emerges upon longer exposures remains to be established, but the trial identified achievable, nontoxic target levels of serum 25(OH)D that may be sufficient to impact inflammatory activity. If, as supported by previous studies and the present trial, vitamin D promotes a shift away from T-cell proliferation and proinflammatory activity, there is reason to hypothesize that vitamin $\mathrm{D}$ could potentially reduce the inflammatory activity complicit in relapses and the neuronal damage implicated in disability and progression. Our results provide preliminary evidence to support studying this hypothesis. Certainly it appears that in the case of MS, a "normal" 25(OH)D level may not be optimal or sufficient to derive benefit at the immunologic level.

Development of a multicenter, randomized, blinded, controlled phase II proof-of-concept trial, powered for MRI and clinical outcomes, is presently underway and is likely the only way to truly define the impact of vitamin D on outcomes meaningful to patients with MS.

\section{AUTHOR CONTRIBUTIONS}

Dr. Burton was the principal investigator of this trial, the attending neurologist who assessed all patients, and performed statistical analyses as well as authoring the present paper. Dr. O'Connor was Dr. Burton's fellowship supervisor from 2005-2007 and assisted as co-principal investigator at that time. Samantha Kimball coordinated patient visits, collected and stored samples, performed sample analyses, and contributed to the statistical analyses. Dr. Burton, Ms. Kimball, Dr. O'Connor, Dr. Vieth, Dr. Bar-Or, Dr. Dosch, and Dr. Ursell all contributed to the trial design. Mr. Cheung performed and participated in the analysis of lymphocyte proliferation assays. Ms. Kimball and Mr. Gagne performed and participated in cytokine analyses. Dr. D'Souza assisted with the protocol for MMP-9/ TIMP-1 assays. All authors discussed the results and reviewed and edited the manuscript.

\section{DISCLOSURE}

Dr. Burton has served on a scientific advisory board for Biogen Idec; has received funding for travel from Biogen Idec, EMD Serono Canada Inc., and Teva Pharmaceutical Industries Ltd.; and has received speaker honoraria from EMD Serono Canada Inc., Teva Pharmaceutical Industries Ltd. and Biogen Idec. Ms. Kimball has served on a scientific advisory board for Actelion Pharmaceuticals Ltd. and received speaker honoraria from Teva Pharmaceutical Industries Ltd. Dr. Vieth has received speaker honoraria from DiaSorin Inc., Merck Serono, Stiefel Laboratories, Inc., and Carlson Laboratories; has been paid by Wyeth to write an article in a newsletter; has served as a media representative for Yoplait Yoghurt; is related to a person employed in the dietary supplement industry; has received research support from the Dairy Farmers of Canada, the National Cancer Institute of Canada, and from the Multiple Sclerosis Society, Canada. Dr. Bar-Or serves on scientific advisory boards for DioGenix, Inc., Ono Pharmaceutical Co. Ltd., and Roche; serves on the editorial board of Neurology; has received speaker honoraria from Biogen Idec, Bayhill Therapeutics, Bayer Schering Pharma (Berlex), Eli Lilly and Company, Genentech, Inc., GlaxoSmithKline, Merck Serono, Novartis, Wyeth, and Teva Pharmaceutical Industries Ltd.; and receives research support from Biogen Idec, Genentech, Inc., Teva Pharmaceutical Industries Ltd., BioMS Medical, the Canadian Institutes of Health Research (CIHR), the MS Society of Canada, The Research Foundation of the MS Society, The Wadsworth Foundation, and the NIH/Immune Tolerance Network. Dr. Dosch founded and serves as President of Afference Therapeutics Inc., in which he holds stock; and receives research support from NIH/NIDDK/TrialNet. Mr. Cheung and Mr. Gagne report no disclosures. Dr. D’Souza received a postdoctoral fellowship from the Canadian Institutes of Health. Dr. Ursell has served on scientific advisory boards for Biogen Idec, Elan Corporation, and Bayer Schering Pharma; has received speaker honoraria from Biogen Idec and Teva Pharmaceutical Industries Ltd.; has received funding for travel from Biogen Idec and Elan Corporation; and receives research support from Biogen Idec and Elan Corporation. Dr. O'Connor serves on scientific advisory boards for Novartis, sanofi-aventis, Bayer Schering Pharma, Genentech Inc., and Roche; has received funding for travel from Biogen Idec and Teva Pharmaceutical Industries Ltd.; has received speaker honoraria from Biogen Idec and Novartis; has served as a consultant for Biogen Idec, Bayer Schering Pharma, Merck Serono, Teva Pharmaceutical Industries Ltd., Genentech Inc., and Warburg Pincus; and receives research support from Bayer Schering Pharma, Novartis, BioMS Medical, sanofi-aventis, and Roche; and serves as National Scientific and Clinical Advisor to the MS Society of Canada.

Received October 9, 2009. Accepted in final form February 3, 2010.

\section{REFERENCES}

1. Ulett G. Geographic distribution of multiple sclerosis. Dis Nerv Syst 1948;9:342.

2. Acheson ED, Bachrach CA, Wright FM. Some comments on the relationship of the distribution of multiple sclerosis to latitude, solar radiation and other variables. Acta Psychiatr Neurol Scand 1960;suppl 147:132-147.

3. van der Mei IA, Ponsonby AL, Blizzard L, et al. Regional variation in multiple sclerosis prevalence in Australia and its association with ambient ultraviolet radiation. Neuroepidemiology 2001;20:168-174.

4. van der Mei IA, Ponsonby AL, Blizzard L, et al. Past exposure to sun, skin phenotype, and risk of multiple sclerosis: case-control study. BMJ 2003;327:316.

5. Islam T, Gauderman WJ, Cozen W, et al. Childhood sun exposure influences risk of multiple sclerosis in monozygotic twins. Neurology 2007;69:381-388.

6. Munger KL, Levin LI, Hollis BW, et al. Serum 25hydroxyvitamin D levels and risk of multiple sclerosis. JAMA 2006;296:2832-2838. 
7. Vieth R, Bischoff-Ferrari H, Boucher BJ, et al. The urgent need to recommend an intake of vitamin D that is effective. Am J Clin Nutr 2007;85:649-650.

8. Soilu-Hanninen M, Airas L, Mononen I, et al. 25Hydroxyvitamin D levels in serum at the onset of multiple sclerosis. Mult Scler 2005;11:266-271.

9. Soilu-Hannien M, Laakson M, Laitinen I, et al. A longitudinal study of serum 25-hydroxyvitamin DD and intact PTH levels indicate the importance of vitamin D and calcium homeostasis regulation in multiple sclerosis. J Neurol Neurosurg Psychiatry 2008;79:152-157.

10. Embry AF, Snowdon LR, Vieth R. Vitamin D and seasonal fluctuations of gadolinium-enhancing magnetic resonance imaging lesions in multiple sclerosis. Ann Neurol 2000;48:271-272.

11. Cantorna MT, Humpal-Winter J, DeLuca F. Dietary calcium is a major factor in 1-25-dihydroxycholecalciferol suppression of experimental autoimmune encephalomyelitis in mice. J Nutr 1999;129:1966-1971.

12. Smolders J, Damoiseaux J, Menheere P, et al. Vitamin D as an immune modulator in multiple sclerosis, a review. J Neuroimmunol 2008;194:7-17.

13. Vieth R. Vitamin D supplementation, 25-hydroxyvitamin D concentrations, and safety. Am J Clin Nutr 1999;69:842-856.

14. Kimball SM, Ursell MR, O'Connor P, et al. Safety of vitamin D3 in adults with multiple sclerosis. Am J Clin Nutr 2007;86:645-651.
15. McDonald WI, Compston A, Edan G, et al. Recommended diagnostic criteria for multiple sclerosis: guidelines from the International Panel on the Diagnosis of Multiple Sclerosis. Ann Neurol 2003;50:121-127.

16. Kurtzke JF. Rating neurologic impairment in multiple sclerosis: an expanded disability status scale. Neurology 1983;33:1444-1452.

17. Banwell B, Bar-Or A, Cheung R, et al. Abnormal T-cell reactivities in childhood inflammatory demyelinating disease and type 1 diabetes. Ann Neurol 2008;63:98-111.

18. Fordyce CB, Gagne D, Jalili F, et al. Elevated serum inflammatory markers in post-poliomyelitis syndrome. J Neurol Sci 2008;271:80-86.

19. Wingerchuk DM, Lesaux J, Rice GPA, et al. A pilot study of oral calcitriol (1,25-dihydroxyvitamin D3) for relapsing-remitting multiple sclerosis. J Neurol Neurosurg Psychiatry 2005;76:1294-1296.

20. Goldberg P, Fleming MC, Picard EH. Multiple sclerosis: decreased relapse rate through dietary supplementation with calcium, magnesium and vitamin D. Med Hypoth 1986;21:193-200.

21. Noseworthy JH, Ebers GC, Vandervoot MK, et al. The impact of blinding on the results of a randomized, placebocontrolled multiple sclerosis clinical trial. Neurology 2001; 57(12 suppl 5):S31-35.

\section{m.AAN.com: Put the AAN in the Palm of Your Hand}

AAN.com, the Academy's award-winning site for neurology resources, has gone mobile. Read more about the special mobile website exclusively available for Academy members at www.aan.com/mobile.

Log on to m.aan.com and access your Academy's web resources on the go.

Visit m.aan.com-it's as close as your phone. 